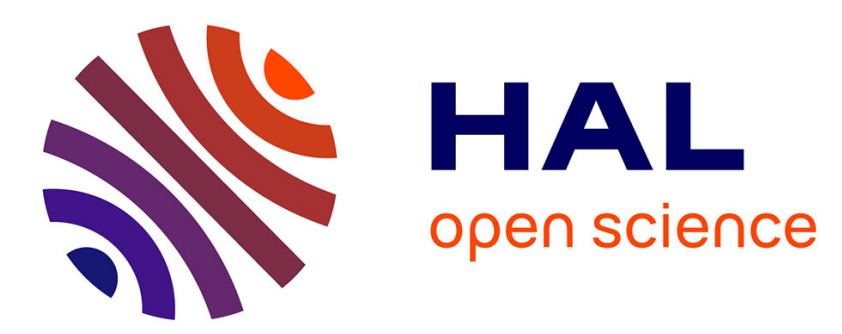

\title{
9. Application des fonctions de bases étendues à l'étude théorique de l'effet tunnel assisté par des impuretés dans les systèmes non uniformes
}

\author{
C.B. Duke, O.G. Kleiman
}

\section{- To cite this version:}

C.B. Duke, O.G. Kleiman. 9. Application des fonctions de bases étendues à l'étude théorique de l'effet tunnel assisté par des impuretés dans les systèmes non uniformes. Revue de Physique Appliquée, 1970, 5 (6), pp.902-903. 10.1051/rphysap:0197000506090203 。 jpa-00243479

HAL Id: jpa-00243479

https://hal.science/jpa-00243479

Submitted on 1 Jan 1970

HAL is a multi-disciplinary open access archive for the deposit and dissemination of scientific research documents, whether they are published or not. The documents may come from teaching and research institutions in France or abroad, or from public or private research centers.
L'archive ouverte pluridisciplinaire HAL, est destinée au dépôt et à la diffusion de documents scientifiques de niveau recherche, publiés ou non, émanant des établissements d'enseignement et de recherche français ou étrangers, des laboratoires publics ou privés. 


\title{
9. APPLICATION DES FONCTIONS DE BASES ÉTENDUES A L'ÉTUDE THÉORIQUE DE L'EFFET TUNNEL ASSISTÉ PAR DES IMPURETÉS DANS LES SYSTĖMES NON UNIFORMES
}

\author{
C. B. DUKE et O. G. KLEIMAN
}

Département de Physique, Materials Research Laboratory and coordinates Sciences Laboratory, Université de l'Illinois à Urbana-Champaign

On établit une théorie microscopique de l'effet tunnel assisté par des impuretés. Dans celle-ci les états propres (étendus) d'un potentiel moyen à un électron dans une jonction tunnel sont utilisés comme fonctions de base. Ces fonctions sont mélangées par l'hamiltonien associé à la présence d'impuretés statiques ou dynamiques en tous points du système. Dans un tel système le propagateur à un électron et le courant correspondant à travers la jonction peuvent être calculés facilement à polarisation nulle par des méthodes classiques [1] à partir des fonctions de Green dépendant de la température. L'existence d'une polarisation finie à travers la jonction est prise en compte dans la théorie par le principe d'occupation rigide; par exemple, l'occupation à l'équilibre des états propres à $N$-particules (qui portent le courant) n'est pas modifiée par la présence d'une polarisation. L'hypo- thèse de l'occupation rigide relie la valeur du courant hors d'équilibre aux propriétés à l'équilibre (polarisation nulle) de la jonction : on obtient de ce fait une théorie du courant hors d'équilibre qui n'est pas basée sur une théorie de la réponse linéaire. L'hypothèse est introduite dans la théorie des perturbations de Matsubara en traitant le potentiel chimique des électrodes du côté gauche et du côté droit comme des multiplicateurs séparés de Lagrange déterminés après avoir effectué toutes les sommes de Matsubara reliées par $\mu_{\mathrm{R}}=\mu_{\mathrm{L}}+\mathrm{eV}$. Donc pour établir et résoudre les équations de Dyson pour les propagateurs renormalisés à un électron, la théorie se réduit au problème classique de l'équilibre défini en utilisant des états d'onde distordus (par exemple des ondes non planes).

En utilisant cette théorie, nous avons construit et résolu les équations de Dyson associées à des fonc- 
tions de vertex «non habillés» pour une impureté statique définie par une fonction delta ou pour une impureté en mouvement elle aussi considérée comme une fonction delta vibrant en un mode local suivant le modèle d'Einstein. Toutes les interactions impuretéélectron (mode de phonons localisés) apparaissent dans ce formalisme comme des corrections de l'énergie propre des fonctions d'onde électronique sur une base étendue. La position de l'impureté par rapport à la région de la jonction détermine le signe et l'amplitude de ces corrections. Les asymétries dans les caractéristiques tunnel peuvent donc être reliées aux positions des impuretés car elles sont prises en compte dans la théorie à l'aide de l'asymétrie de la self énergie (induite par l'impureté) de deux états de base du courant linéai- rement indépendants c'est-à-dire celui de gauche à droite et de droite à gauche.

En utilisant les solutions des équations de Dyson nous avons calculé les caractéristiques tunnel d'une jonction formée d'une barrière carrée en fonction de la position d'un plan d'impuretés défini par une fonction delta. La forme détaillée du profil de la caractéristique dépend à la fois de la position des impuretés et de la forme du potentiel moyen à un électron. Cependantsi on se donne le potentiel moyen on peut obtenir des informations qualitatives concernant la position des impuretés pouvant être déduites des résultats expérimentaux.

[1] Duke, (C. B.) Tunneling in Solids, Academic Press, N. Y., 1969.

\title{
11. MÉCANISMES DE PERTES D'ÉNERGIE DES QUASI-PARTICULES EXCITÉES DANS UN SUPRACONDUCTEUR
}

\author{
C. J. ADKINS et A. R. LONG
}

Royal Society Mond Laboratory, Free School Lane, Cambridge, England 